\title{
Mathematical Modelling - An Effective Method for Making Mathematics Learning Joyful At Lower Secondary Level
}

\author{
Omprakash Mishra \\ Headmaster(i/c),Govt. High School, Jeypore . District: Koraput; State: Odisha; India
}

\begin{abstract}
Since time immemorial mathematics has been considered as a boring subject having no real life connections and implications. However sometimes it is also argued that if properly carried out mathematical problem solving via modelling perspective can focus on the students' representational fluency through the flexible use of mathematical ideas. It is believed that as interpretive cycles take place within the modelling process, multiple mathematical interpretations of students are elicited within each modelling stage. The present paper, where the basic grid models of numbers are used to find out the HCF and LCM in classroom to find out its effectiveness as a method of teaching is actually the theoretical supplement of a workshop. Mathematical modelling helps in transforming as well as linking the mathematical problems into real world situations and hence helps the child enjoy learning mathematics. Mathematical modelling and its applications is a process of solving a particular type of problems generated by corresponding situations of the real world. Nowadays it is generally acceptable that through problem solving processes one can give students a balanced view of mathematics and can face effectively the false opposition between learning mathematics and learning to apply mathematics. The present article adopts a conceptual framework that integrates a range of significant issues that impact on the field of applications and modelling in mathematics education. The focus areas include ability of the teacher to devise models that suits a particular mathematical problem, the impact of task context and perception of students after its implementation particularly in lower secondary class rooms.
\end{abstract}

Keywords: Grid models, HCF , LCM, set intersection, prime factorisation

\section{Introduction}

In India it is a well known fact that many mathematics teachers are not familiar with modeling or do not want to spend time on modeling in mathematics class. Therefore we first address the question what modeling is about and why it should be included in the mathematics curriculum. The application of mathematics cannot be divorced from the use of models and the modeling process. As most application problems involve the discussion of some reality or "realistic contexts", the use of models to access that reality becomes essential.In application problems, children very often have to deal with problems in realistic contexts which can only be made accessible and manipulable if models are used. Thus, models have an important role in making mathematics become real for students ( Kaur \&Dindyal, 2010). The new curriculum now states that applications and modeling "should be part of the learning for all levels" and defines it as "the process of formulating and improving a mathematical model to represent and solve real-world problems".

\subsection{NCF 2005 and mathematics education}

The prime goal of mathematics education emphasized in NCF 2005 is 'mathematization' of the child's thought and processes. However it realized that "language of mathematics learnt in school is far removed from their everyday speech, and easily forbidding" (NCERT, 2006, p. 5). The curriculum therefore also expects that the language used in textbooks would be like that spoken by children in their daily life. Hence it advocates for the development of a mathematics program that would ensure that everybody learns mathematics and does not fear it.

\subsection{Research questions}

Based on the objectives, the following research questions were derived:

- How does the integration of applications and modelling activities impact student's attitude and motivation for learning of mathematical concepts?

- Does introducing mathematical modelling activities, help teachers motivating and teaching mathematical concepts?

- Does modelling activities in the class affect student's interest towards mathematical tasks? 


\section{Back ground of the study}

The present study that I am pursuing is part of a larger study in investigating the perception, attitude and inerest of teachers as well as students of lower secondary $8^{\text {th }}$ class in mathematical modelling process in tribal areas of Koraput district. This study adopts a conceptual framework that integrates the modelling approach into a problem-based learning or instructional setting. The problem-based learning setting is to highlight the teachers role in a modeling approach, and the student collaboration. The task is also specifically designed based on principles that are aligned with reformed classroom practices that include a modelling slant. Model-eliciting task would provide the context for argumentation and collaboration, and the teacher functions as a facilitator to provide guidance at certain junctures of the modelling process. The pupils' cognitive processing is manifested through the modelling stages of Description, Manipulation, Prediction and Optimisation. Here, Description refers to attempts at understanding the problem to simplifying it . Prediction refers to the scrutinising of the models that the students have conceived as they analyse the designs or solutions towards ensuring that they fit the parameters given or established. Optimisation refers to extending the models .

\section{Methodology and Procedure}

The method adopted was qualitative only. In this article I have described an alternative representation of whole numbers, that can be constructed as a manipulative model and has been used by the teachers to teach the process to help finding the Greatest Common Factor and the Least Common Multiple of numbers.

\subsection{Participants and grouping}

For this , 8th class students of two upgraded high schools were selected for the study. A total of 40 students were divided into five small groups. The two main criteria for the assignment of group members were that each member should feel comfortable enough with their friends to negotiate and communicate their ideas, and that the groups should be heterogeneous in terms of mathematical ability. The selection was based on the teachers knowledge about the student and the records of last examination marks. Also 5 teachers of the respective schools who are teaching mathematics to the above students were selected for the study to transact the modeling classes.

\subsection{Research tools}

The study included diverse research instruments for the various populations that participated. The following measures were included:

1. Structured interview schedules for teachers: After the modeling class transactions are over the teachers were asked about the difficulties they faced in teaching while providing different mathematical concepts. These teachers were asked about the impact of the project on the school and the students, attitudes regarding learning and instruction with the models, major difficulties and advantages of the project. Their perception about teaching mathematics through model making.

2. Structured interview schedules for students: The students were asked about their prior knowledge of model making and its use and their attitudes toward learning via the model making, their interest and motivation to learn, relevance of the content, engagement in class discussions, group work, evaluation of achievements, and command of learning skills. Also the students were asked about the differences between regular lessons versus lessons through model making.

3. Interview with the head of the Schools: An interview schedule was administered to the Heads of the two school where the study was conducted to know about their perception and attitude towards the use of models during mathematics teaching.

\subsection{The educational setting}

Normally the school teachers while teaching on number theory, HCF and LCM follow the traditional approaches like , 1- Set intersection: creating an ordered list of consecutive multiples for each number and finding the first one that appears in both lists ; 2. Creating a multiple and divide: creating an ordered list of consecutive multiples of one number and simultaneously checking each new entry for divisibility by the second number; 3. Prime-factorization: comparing the prime factorization of the two numbers and finding the minimal product of prime powers that contains both of their factorizations (often referred to as "the higher exponent" rule) to find the answers.

\subsubsection{Preparing the teacher-facilitators for the study}

The mathematics teachers of the respective classes participated as teacher facilitators. However most of the teachers expressed their difficulties to handle a model eliciting mathematics class room as they have never done this before as well as never being trained about this. Hence, Prior to the modelling session, the teacher facilitators were brought through a facilitation training session with the author. The teacher facilitators had to 
conduct a modelling session on finding the HCF and LCM of two numbers in the class through a grid model so that the students can understand the concept of HCF and LCM and work the problem based on it while working in small collaborative groups.

\subsection{Grid models of whole numbers}

This kind of manipulative model, which I am referring as grid model, was proposed by Grossi ( 2000), and was based on Hasse diagrams. To build these models you will need some thermocool spheres and wooden sticks painted

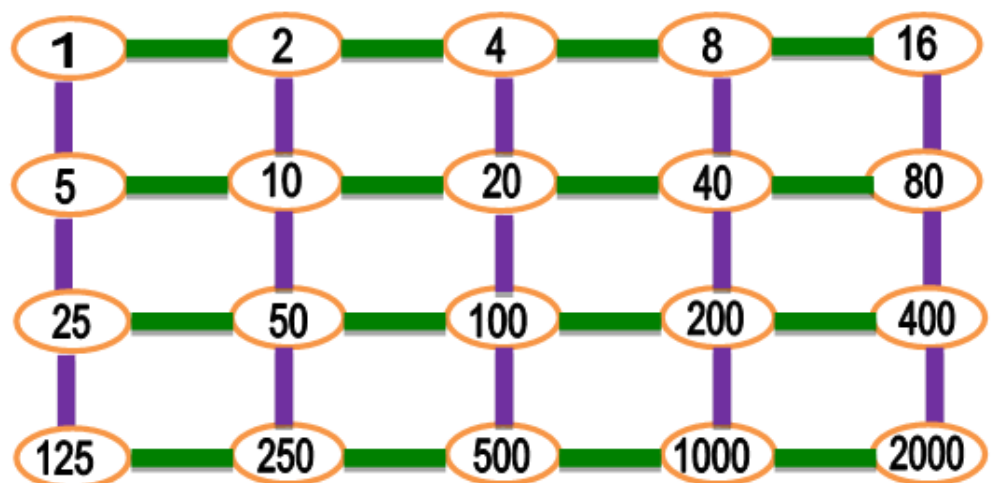

Figure 1 grid model for the number, $2000=2^{4} \times 5^{3}$

in different colors or steel wires wrapped with different colored straws. The first thing to be done to construct the models is to choose a different color to represent each prime number that is going to be represented. For example, we can assume that the color green will represent the prime 2, yellow will represent 3, purple will represent 5, black will represent 7 , and so on. The wooden sticks should be painted or the straws should be in these colors, and they will represent the prime factors in the constitution of a number. The thermocool spheres will represent the factors of a number, and will be labeled with a pen or marker. Let us look at this example: If the number we want to model is 2000 which is equal to $2^{4} \times 5^{3}$, we start by getting 4 sticks of the color that represents the prime 2 and placing them in sequence. At the extremeties of each stick, we attach a thermocool sphere labeled with the sequence $2^{0}=1,2^{1}=2,2^{2}=4,2^{3}=8$, and $2^{4}=16$. We then prepare another linear sequence of spheres and sticks for the sequence $5^{1}=5,5^{2}=25,5^{3}=125$ (we may not write the factor 1 because we have already labeled a sphere as ' 1 ' in the previous sequence).

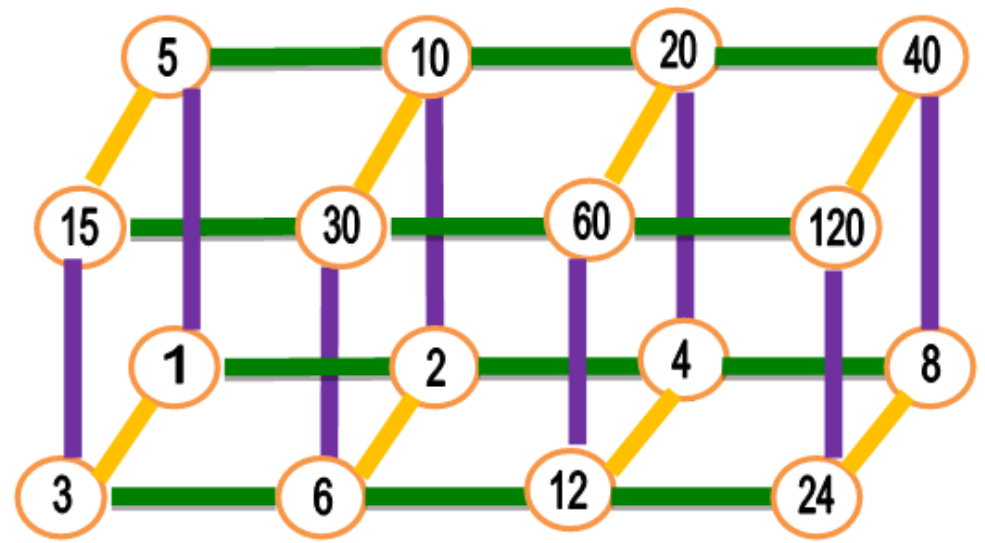

Figure2 model having three distinct primes as their factors

Now we attach it to the previous sequence, by pinning or attaching it perpendicularly at the sphere labeled "1". We continue by filling up the sequence to represent the products of the powers of 2 by the powers of 5. The resulting model would look like as shown in Fig.-1.

So, for example, in the configuration in Fig. -1 , the green stick represents the operation "times 2" if read from left to right, and "divided by two" if read from right to left. In other words, the green stick represents the prime two (because we have choosen green to represent 2) as an operator in a multiplication or in a division. Similarly, purple sticks would represent "times five" or "divided by five", depending if you are moving from top to bottom or from bottom to top, respectively, in the diagram. Fig.-2 show models of numbers that have three 
distinct primes as their factors, respectively. All these grid models reflect all the characteristic properties of positive integers, including the uniqueness of their prime factorization: Since no two numbers have the same prime factorization - hence no two numbers will have two equal grid models.

\subsection{Finding the GCF and LCM using the grid models}

When we look for the Greatest Common Factor (GCF) we have to compare the set of factors of those numbers and find which factors they have in common, and then, among those, which one is the greatest.

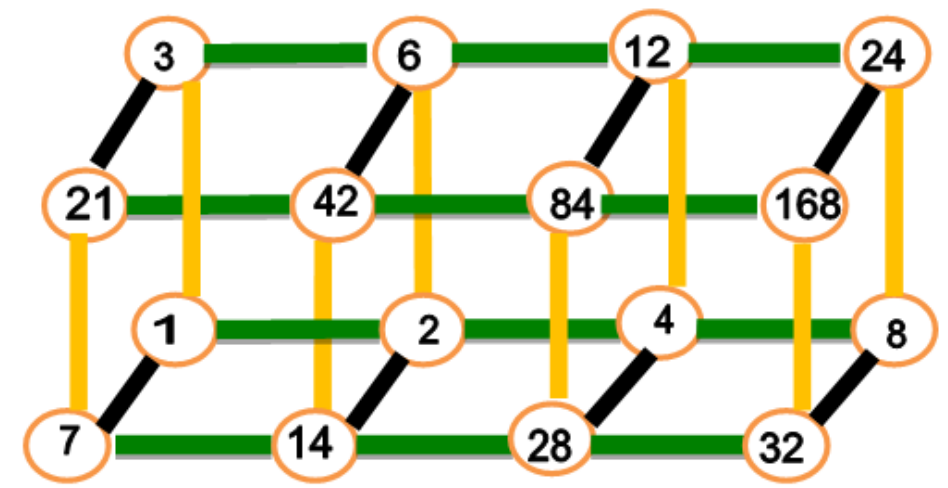

Figure 3 Grid models for the numbers 168 and 588

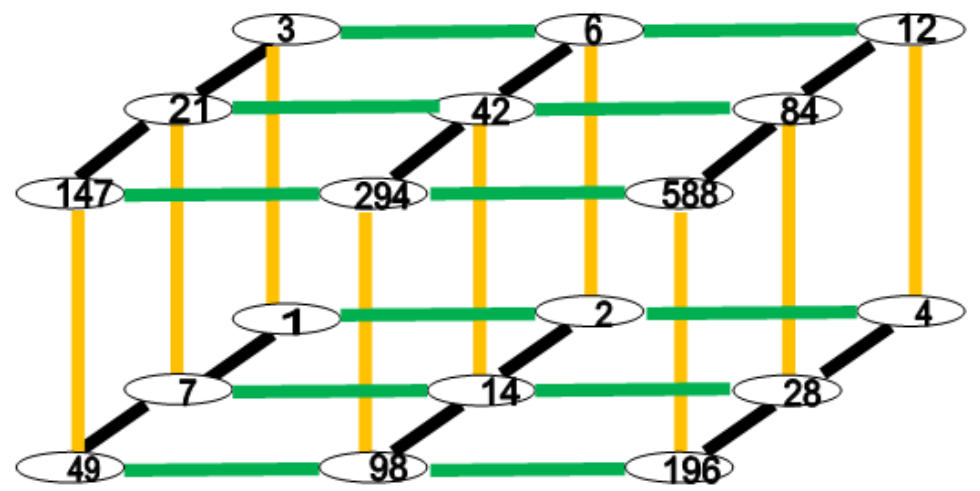

Figure 4 Grid models for the numbers 168 and 588

This grid model gives us the factors of numbers. So we can easily compare two grids and see the greatest common factor in those grids For example, here are the models for the numbers 168 and 588, shown in Fig. -3 and Fig.-4, we can see that the intersection between the two sets of factors or the part of the two models that have in common is the one whose thermocool balls were shaded purple. Since those are the common factors, the greatest common factor is the greatest among them, that is 84 . It may be noted that 84 is in the opposite corner from the number which one is the shaded common part of the models.
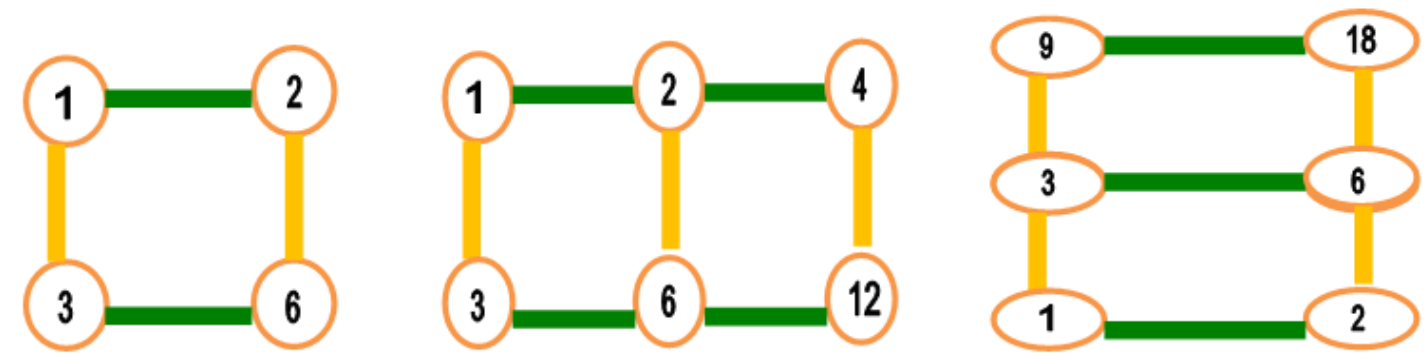

Figure 5 The model for the number 6 is part of the models for the numbers 12 and 18

Now coming to the use of the grid models to find out the Least Common Multiple of two or more numbers we know that a multiple of a number $\mathrm{n}$ is any number whose grid model includes the grid of $\mathrm{n}$. For example, since the grid for 12 includes the grid for 6 , we know 12 is a multiple of 6 . Since the grid for 18 also includes the grid for 6, the number 18 is another multiple of 6 (Fig.-5). 


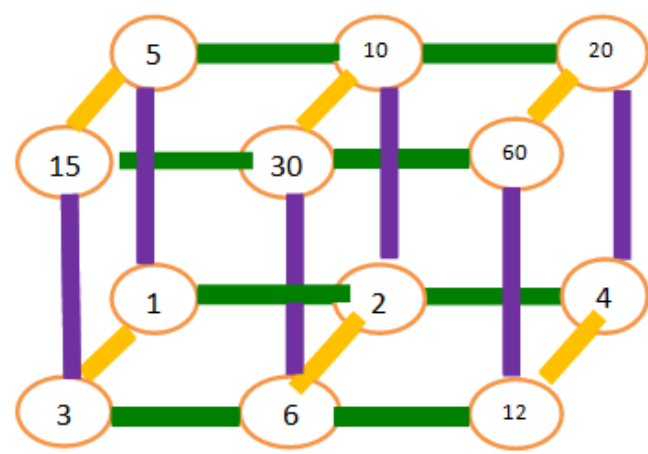

Figure 6 (a) Grid model for 60

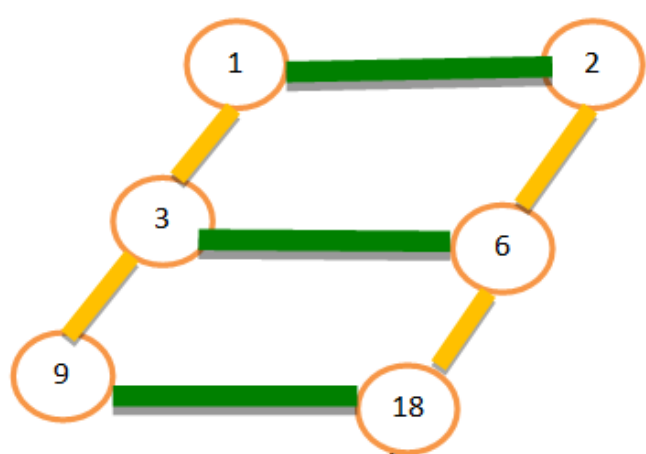

Figure 6 (b) Grid model for 18

We see that, while we can see the factors of a number ' $n$ ' in its grid model, to get a multiple of ' $n$ ' we would have to construct another model - one of which the model for ' $n$ ' is a part. So when we look for the least common multiple between two or more numbers, we have to look for a number that we have to construct, in whose grid we will find all the original numbers (that is, the numbers whose LCM we are going to find out). And among all the possible common multiples of these numbers, or all the models that can be built containing these original models as parts, which one is the least ? In other words, what is the smallest model we can build which contains those numbers whose LCM we are trying to determine ? Let us examine, for an example, the process of finding the LCM of 18 and 60. Their models are shown in Fig. 6 ( a) and 6 ( b). The model for the LCM will have to include both these models among its parts, because both 18 and 60 are factors of the LCM. If we compare the two models, we see that the model for 60 has one more power of 5, and the model for 18 has one more power of 3. Fig. 6 ( c) shows the two models combined, but in incomplete form- that is, the set form is not a model for any number. We then complete the grid to find the smallest model that will contain that combined set, finding the model for 180, shown in Fig. 6 (d).

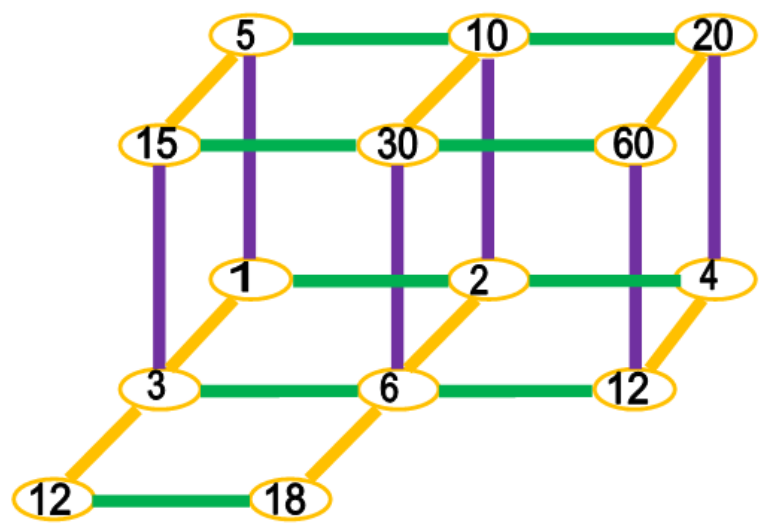

Figure 6 (c) An incomplete model

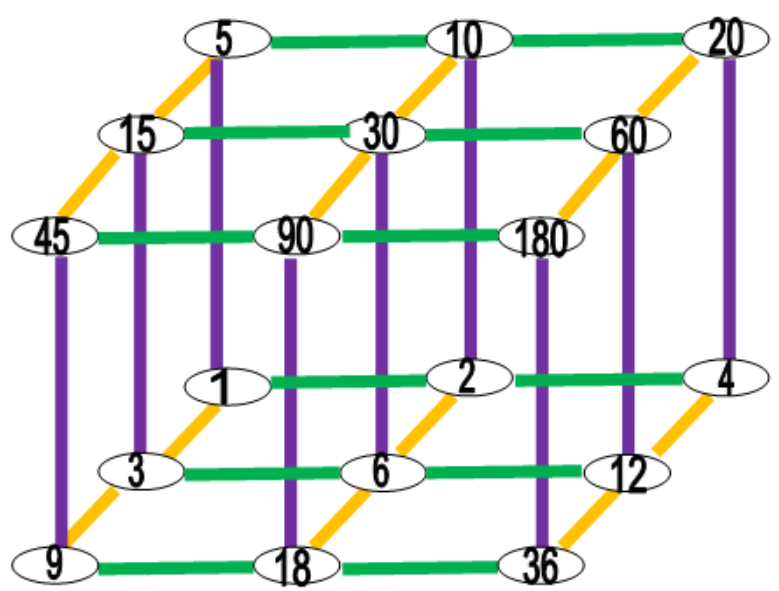

Figure 6 (d) The complete model for the LCM combining the models for 60 and 18 


\section{Analysis and Result}

The data collected through structured interview schedules from the key informants such as students and teachers were analysed with the help of percentages after the class room transactions were over. These data, however, were supplemented and substantiated with the help of the data collected through different qualitative tools such as focus group discussions, interview and observation.

TABLE-1 clearly indicates that about $92.5 \%$ of the students enjoyed learning through modeling approach and hence advocated more classes through this approach. 85\% students felt that they are motivated for learning mathematics and could understand the concept of HCF \& LCM, further the same amount prefered the modeling approach over the traditional ones . $70 \%$ of the learner felt that mathematics is a easy subject and other mathematics topics can also be taught in this way. Interestingly a $15 \%$ of the students were not sure about it . $87.5 \%$ of students were of the view that studying through modeling is easier than the traditional approach and an equal percentage also opined that learning in groups was enjoyable. $95 \%$ students agreed to the appropriateness of the model for the learned topic and $97.5 \%$ believed that the materials needed for the particular model were within their reach.

Table 1 Students' Attitudes and interest towards Learning through modelling

\begin{tabular}{|c|c|c|c|c|}
\hline \multirow{2}{*}{$\begin{array}{l}\text { Sl. } \\
\text { No }\end{array}$} & \multirow[t]{2}{*}{ Key indicators } & \multicolumn{3}{|c|}{ Percentage of respondents } \\
\hline & & Yes & No & Can't say \\
\hline 1 & Enjoy learning & 92.5 & 7.5 & 0 \\
\hline 2 & motivated for learning & 85 & 10 & 5 \\
\hline 3 & can understand the learning materials & 85 & 15 & 0 \\
\hline 4 & Mathematics is easy & 70 & 27.5 & 2.5 \\
\hline 5 & Studies through modeling are easier & 87.5 & 7.5 & 5 \\
\hline 6 & Prefer modeling approach over traditional one & 85 & 10 & 5 \\
\hline 7 & The teacher helps students in model making & 90 & 10 & 0 \\
\hline 8 & Appropriate model for learning HCF \&LCM & 95 & 5 & 0 \\
\hline 9 & working in groups is enjoying & 87.5 & 12.5 & 0 \\
\hline 10 & Want more classes on model making & 92.5 & 7.5 & 0 \\
\hline 11 & Other mathematics topics should be taught through modelling & 70 & 15 & 15 \\
\hline
\end{tabular}

TABLE-2 here, regarding the attitude, interest and perception of the teachers towards teaching through modeling approach clearly indicates that all most all the mathematics teachers $(100 \%)$ involved in the process enjoyed teaching and they admitted that it helps the students in collaborative and cooperative learning, also the students appreciate the teachers more in this approach. However they all advocated for special teacher training programmes on math-modelling . $80 \%$ of the teachers expressed their difficulties in planning appropriate models for specific topics, though they felt that this is a credible way to clear mathematical concepts as the students are getting motivated to learn mathematics through this approach. The same $80 \%$ of the teachers preferred modeling approach to the traditional one. $60 \%$ of the teachers believe that learning goals can be achieved through modeling approach, however it is interesting to note that $40 \%$ of the teachers are not sure about it. Again $60 \%$ felt that the present curriculum supports the modeling approach and they opined that adopting the approach does not create any extra burden on them .

TABLE 2 Teachers' Attitudes and interest for a math-modeling classroom

\begin{tabular}{|c|c|c|c|c|}
\hline \multirow{2}{*}{ Sl. No } & Key indicators & \multicolumn{3}{|c|}{ Percentage of respondents } \\
\cline { 2 - 5 } & & Yes & No & Can't say \\
\hline 1 & enjoyed teaching & 100 & 0 & 0 \\
\hline 2 & It involves extra burden & 40 & 60 & 0 \\
\hline 3 & Difficult to plan appropriate models for different topics & 80 & 20 & 0 \\
\hline 4 & Students understanding of the concepts is easy & 80 & 20 & 0 \\
\hline 5 & Model making approach helps collaborative learning & 100 & 0 & 0 \\
\hline 6 & Students are motivated to learn mathematics & 80 & 0 & 20 \\
\hline 7 & Present curriculum supports this approach & 60 & 20 & 20 \\
\hline 8 & Was it an appropriate model to teach HCF \& LCM & 100 & 0 & 0 \\
\hline 9 & learning goals can be easily achieved & 60 & 0 & 40 \\
\hline 10 & Training on model making is essential & 100 & 0 & 0 \\
\hline 11 & students appreciate the teacher more & 100 & 0 & 0 \\
\hline 13 & Like to prefer modeling approach to the traditional one & 80 & 20 & 0 \\
\hline
\end{tabular}

The head teacher of the two schools whose students participated in the study when interviewed by the author clearly appreciated the classroom environment during teaching mathematics through modelling . They also advocated for more training programmes for the mathematics teachers, for a full fledged implemention of the approach in their schools. Further they suggested provisions for math-modeling in curriculum. 


\section{Suggestions and Conclusion}

The application of mathematics cannot be divorced from the use of models and the modelling process. As most application problems involve the discussion of some reality or "realistic contexts", the use of models to access that reality becomes essential. In application problems models have an important role in making mathematics become real for students. The most important benefit that can be extracted through this approach is that it will change the perception of the students about mathematics as an entertaining subject rather than a boring one. However it must be noted that use of modeling is not a magic bullet, but a tool with tremendous capabilities, particularly when physical manipulatives are combined with matching virtual manipulatives to engage students in shared mathematics thinking and discourse. In order to achieve the goal of a modelling class room it is suggested that we have to include applications and modelling topics more centrally within the curricula . Also sufficient pre-service and inservice practical training programmes on mathematics modelling class rooms are to be provisioned as an integral part of the training syllabus of the pre-service teachers.

\section{References}

[1]. Werner Blum, University of Kassel, blum@mathematik.uni-kassel.de ,Rita Borromeo Ferri ,University of Hamburg

[2]. borromeo@erzwiss.uni-hamburg.de, Mathematical Modelling: Can It Be Taught And Learnt? Journal of Mathematical Modelling and Application ,2009, Vol. 1, No. 1, 45-58

[3]. Ana Dias.Central Michigan University, dias1al@cmich.edu, Using lattice models to determine Greatest Common Factor and Least Common Multiple : pages 3-8

[4]. Brown, Anne. (2002) 'Patterns of thought and prime factorization', in Learning and teaching number theory: Research in cognition and instruction, edited by Stephen Campbell and Rina Zazkis, 131-137. Westport: Ablex publishing.

[5]. 'position paper national focus group on teaching of mathematics', National Council of Educational Research and Training, New Delhi, India, First Edition Mach 2006.

[6]. Zazkis R. \& Campbell, S. (1996) "Divisibility and muliplicative structure of natural numbers: Preservice teachers' understanding. Journal for Research in Mathematics Education, 27(5), 540-563. 